\title{
Ampliación del tamizaje de errores innatos del metabolismo en Perú: reporte de caso con trastorno del metabolismo de cobalamina
}

\section{Widened screening for innate metabolism disorders in Peru: report of a case with cobalamin metabolism disorder}

Correspondencia Hugo Hernán Abarca Barriga habarca@insn.gob.pe

Recibido: 01/02/2020 Arbitrado por pares Aprobado: 04/03/2020

Citar como: Abarca-Barriga HH, Rodríguez RS. Ampliación del tamizaje de errores innatos del metabolismo en Perú: reporte de caso con trastorno del metabolismo de cobalamina. Acta Med Peru. 2020;37(1):78-83. doi: https://doi. org/10.35663/amp.2020.371.880
Hugo Hernán Abarca-Barriga ${ }^{1,2,3, a}$, Richard S. Rodriguez $z^{4, b}$

Servicio de Genética y Errores Innatos del Metabolismo, Instituto Nacional de Salud del Niño. Lima, Perú.

Carrera Profesional de Medicina Humana, Universidad Científica del Sur. Lima, Perú.

Facultad de Medicina Humana, Universidad Ricardo Palma. Lima, Perú.

Equipo Funcional de Genética y Biología Molecular, Instituto Nacional de Enfermedades Neoplásicas. Lima, Perú.

Médico genetista, magíster en Genética; ${ }^{\mathrm{b}}$ médico cirujano.

\section{RESUMEN}

El tamizaje neonatal de los errores innatos del metabolismo se instauró hace más de 50 años en el mundo. En Latinoamérica, Uruguay, Costa Rica, Chile, Brasil y Colombia han implementado esta política de salud pública de manera sostenida. La tecnología para detectar estas enfermedades ha ido progresando con un mejor costo/efectividad, haciendo que sea de acceso casi universal. Los trastornos del metabolismo intracelular de la cobalamina es un grupo heterogéneo clasificados en tres fenotipos bioquímicos. Reportamos al primer paciente en Perú con diagnóstico tardío de una variante homocigota c.394 C>T en el gen MMACHC, el cual pertenece al grupo de complementación cblC el cual produce aciduria metilmalónica y homocistinuria, caracterizado por talla baja, hipotonía, retraso del desarrollo psicomotor, convulsiones, anemia megaloblástica, trombocitopenia y neutropenia ondulantes; con homocisteína elevada, acidemia metilmalónica, y contradictoriamente aumento de vitamina B12 en sangre. Es importante el diagnóstico oportuno de enfermedades potencialmente tratables, evitando o disminuyendo la severidad del fenotipo, a través de la implementación de nuevas tecnologías en nuestro país.

Palabras clave: Errores innatos del metabolismo; Cobalamina; Tamizaje neonatal; Perú (fuente: DeCS-BIREME). 


\section{ABSTRACT}

Neonatal screening for innate metabolism disorders was instituted more than 50 years ago. In Latin America, countries like Uruguay, Costa Rica, Chile, Brazil, and Colombia have implemented this public health measurement in a sustained fashion. Technology for detecting these conditions has been steadily progressing, achieving a good cost/effectiveness ratio, so access for such test is practically universal. Intracellular cobalamin metabolism disorders constitute a heterogeneous group that is subdivided in three biochemical phenotypes. We report the first patient in Peru with a late diagnosis of a homozygous c.394 C>T variant in the MMACHC gene, which belongs to the cbIC complementation group, which leads to methyl-malonic aciduria and homocystinuria, characterized by low height, retardation of psychomotor development, seizures, megaloblastic anemia, and variable thrombocytopenia and neutropenia. Also, homocysteine levels are high, there is methyl-malonic academia, and there is a paradoxical vitamin B12 increase in peripheral blood. This paper emphasizes the importance of making a timely diagnosis of potentially treatable conditions, avoiding or reducing the severity of the implied phenotype, with the implementation of new technologies in our country.

Keywords: Inborn errors metabolism; Cobalamin; Neonatal screening; Peru (source: MeSH NLM).

\section{INTRODUCCIÓN}

El término de error innato del metabolismo (EIM) fue introducido en el año 1909 por Sir Archibald Garrod, quien describió a la alcaptonuria, albinismo cistinuria y pentosuria; incorporando la idea de la «individualidad bioquímica» ${ }^{[1]}$. Los EIM son un grupo de enfermedades que tienen una prevalencia de 1 por cada 1000 personas ${ }^{[2]}$. El número de EIM conocidos a la fecha son aproximadamente 1015 entidades bien definidas, dentro de las cuales se encuentran los trastornos intracelulares de la cobalamina ${ }^{[3]}$.

La cobalamina es convertida en dos coenzimas: metilcobalamina (MeCbl) y adenosilcobalamina (AdoCbl). La MeCbl es utilizada para la conversión de homocisteína a metionina, a través de la metionina sintasa; mientras que la AdoCbl es utilizada por la enzima mitocondrial metilmalonil CoA mutasa, la cual convierte a la L-metilmalonil-CoA a succinil-CoA ${ }^{[4]}$. Los trastornos intracelulares de la cobalamina (TIC) son clasificados en nueve grupos de complementación genética (cblA-G, JyX) ${ }^{[5]}$. La aciduria metilmalónica y homocistinuria tipo cbIC o ACMMHC (MIM \#277400) es el trastorno más común dentro de los TIC, el cual es provocado por variantes en el gen $M M A C H C$, localizado en $1 \mathrm{p} 34.1^{[4]}$, causando una disminución de AdoCbl, ni MeCbl, lo que se manifiesta clínicamente por dificultades en la alimentación, fallo de medro, trastornos hematológicos, neurológicos, renales, oftalmológicos y dermatológicos ${ }^{[6]}$. Otras manifestaciones son hidrops fetalis ${ }^{[7]}$, retraso del crecimiento intrauterino, anomalías cardíacas ${ }^{\left[{ }^{[8]}\right.}$, cardiomiopatía dilatada ${ }^{[9]}$, esteatosis hepática, enteropatía perdedora de proteínas y linfohistiocitosis hemafagocítica ${ }^{[10]}$. Dentro de las manifestaciones bioquímicas son vitamina B12 dentro de valores normales, homocistinuria, homocistinemia, aciduria metilmalónica, metionina disminuida, cistationemia y rara vez hiperamonemia con acidosis metabólica ${ }^{[7]}$.

La enfermedad por cblC se clasifica según las características clínicas en fenotipo severo y moderado. El primero con manifestaciones sistémicas desde el primer año de vida (neurológicas, hematológicas y oftalmológicas) y una mortalidad de la cuarta parte; $y$ el segundo con manifestaciones extrapiramidales, demencia, delirio o psicosis ${ }^{[11]}$. El tratamiento en ambos grupos es con hidroxicobalamina, betaína, ácido fólico y carnitina ${ }^{[10,11]}$. Algunos pacientes, a pesar del tratamiento, podrían desarrollar severas complicaciones; más aún si el tratamiento no fue oportuno ${ }^{[10]}$. La efectividad de la cobalamina parenteral se ve respaldada por la mayor supervivencia de los pacientes que desarrollan el síndrome urémico-hemolítico ${ }^{[12]}$ y la reversión de complicaciones graves ${ }^{[10]}$. El uso de dosis más altas de cobalamina parenteral ha sido eficaz para reducir los metabolitos y ha llevado a la resolución de la microangiopatía trombótica en pacientes con enfermedad por cblC ${ }^{[13]}$. La incidencia de la enfermedad por cblC es variable según las poblaciones y en Perú se desconoce la incidencia de esta entidad ${ }^{[10]}$.

Se describe el primer paciente por deficiencia de cblC en Perú, haciendo hincapié en la historia natural de la enfermedad hasta los dos años y seis meses de edad; y luego como muchos de los síntomas y signos se revierten progresivamente, asimismo, se plantea la importancia del tamizaje neonatal ampliado.

\section{REPORTE DE CASO}

Paciente varón de dos años seis meses, nacido de parto vaginal a término, con peso al nacer de $2735 \mathrm{~kg}$, talla de $50 \mathrm{~cm}$, perímetro cefálico desconcido y con puntaje de Apgar de $8^{1}-9^{5}$. Tiene una hermana mayor sin antecedentes médicos y los padres son consanguíneos ( $\mathrm{F}=1 / 8$ ) (Figura $1 \mathrm{~A})$. La antropometría para la talla, perímetro cefálico y peso se encuentra en los percentiles de 57,13 y 15 respectivamente; su desarrollo psicomotor tuvo un retraso, observándose que el control cefálico fue al año, control torácico con apoyo al año seis meses, y sonrisa social al año un mes. Al examen físico no se observan dismorfias, pero presenta pelo ralo, livedo reticularis, tono muscular disminuido a predominio axial.

Presentó varias hospitalizaciones, al nacer por ictericia, a los 13 días de vida por neutropenia severa, a los siete meses por otitis media aguda y neutropenia; 11 meses por epilepsia; un año 

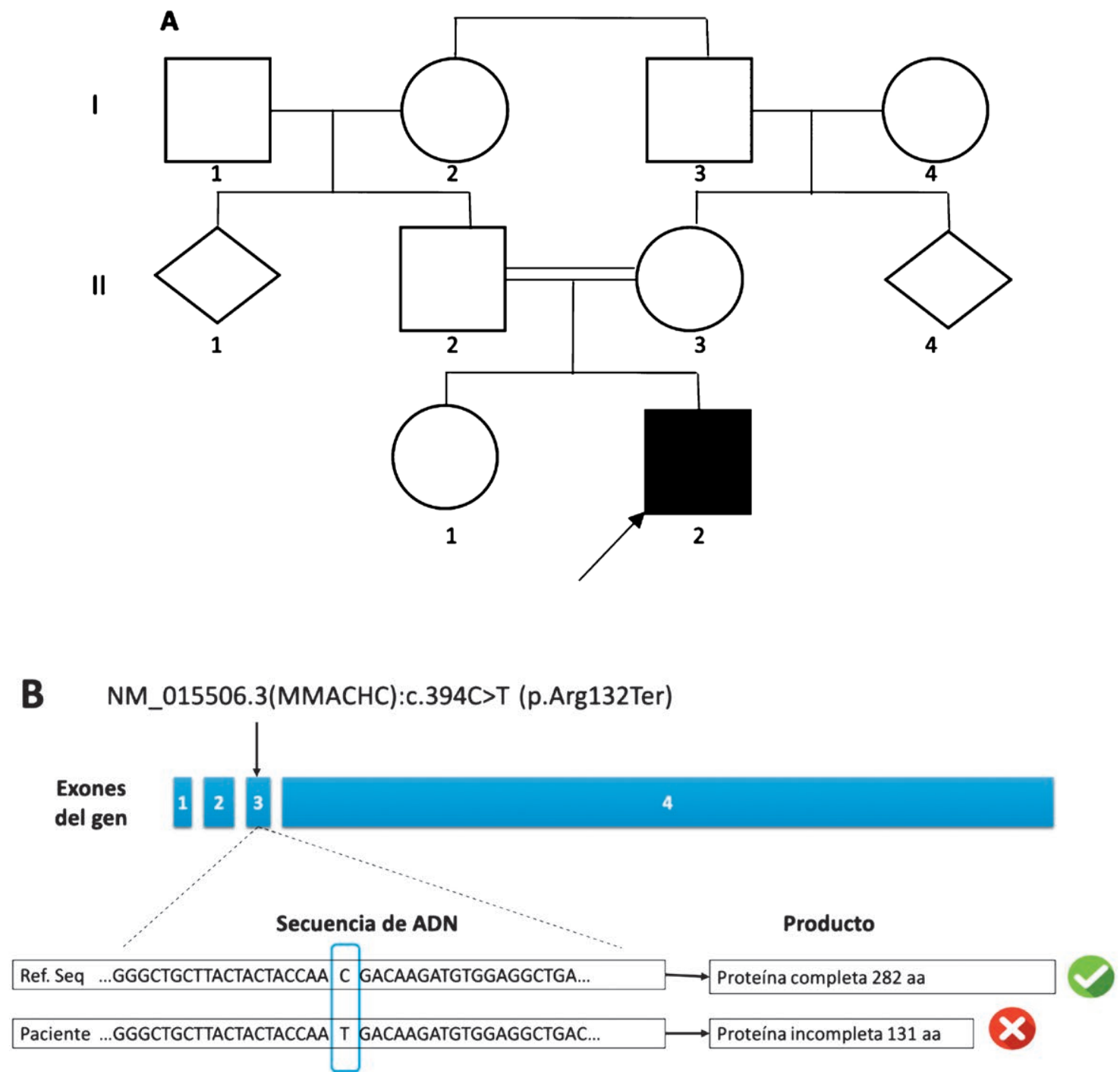

Figura 1. A. Árbol genealógico, nótese que los padres (II.2 y II.3) del propositus (III.1) tienen un coeficiente de endogamia (F) de 1/8. B. Impacto clínico de la variante patogénica del gen MMACHC. Ubicación de la variante patogénica c.394C $>$ T (p.Arg132Ter) en el gen $M M A C H C$ y la consecuente producción de una proteína truncada con alteración de función; en azul: exones del gen $M M A C H C$ (secuencia de referencia del ADN normal).

dos meses por epilepsia, pancitopenia, impétigo, estomatitis aftosa. Para cada episodio de neutropenia recibió filgastrin, no observándose una respuesta favorable.

Los exámenes complementarios a los 11 meses de edad se observan en la Tabla 1. Por los resultados alterados, se realizó la determinación de variantes patogénicas de la acidemia propiónica (E168K, 1218del, 14/ins 12, 1170 insT) y de la acidemia metilmalónica (N129Y, G717V), no observándose cambios. Los exámenes que estuvieron dentro de la normalidad fueron los potenciales evocados auditivos, electroencefalograma, perfil tiroideo, cariotipo 46,XY; citometría de flujo de CD 19+, CD3+, CD4+, CD8+, CD16/56+, ecografía renal y hepática. En la resonancia magnética de encéfalo se observa hipotrofia cerebral de lóbulos frontales y temporales, retraso de la mielinización de la sustancia blanca.
El secuenciamiento exómico clínico encontró una variante homocigota en el gen MMACHC en c.394 C>T, el cual provoca un codón de parada (Figura 1B), por lo que fue clasificada como patogénica y está disponible en la base de datos ClinVar (VCV000001423.6) con la nomenclatura NM_015506.3(MMACHC):c.394C>T (p.Arg132Ter); no se encontró otras variantes patogénicas incidentales.

Se inició tratamiento al año cinco meses con hidroxicobalamina a dosis de $1 \mathrm{mg}$ intramuscular diario y de ácido fólico de 0,5 mg diarios. Un control a los cuatro meses de iniciado el tratamiento, se observó una disminución en plasma de homocisteína y persistencia de vitamina B12 alta (Tabla 1). En el último control a los dos años y tres meses, el hemograma estaba dentro de parámetros normales. 
Tabla 1. Principales resultados de laboratorio basal y postratamiento.

Pruebas de laboratorio Valor Valor normal

Basales

\begin{tabular}{lcc}
$\begin{array}{l}\text { Sangre } \\
\text { C3* }\end{array}$ & $4,15 \mu \mathrm{mol} / \mathrm{L}$ & $<3 \mu \mathrm{mol} / \mathrm{L}$ \\
C3/C2* & $0,6 \mu \mathrm{mol} / \mathrm{L}$ & $<0,3 \mu \mathrm{mol} / \mathrm{L}$ \\
C3/C16* & $8,74 \mu \mathrm{mol} / \mathrm{L}$ & $<2,2 \mu \mathrm{mol} / \mathrm{L}$ \\
Vitamina B12† & $1104 \mathrm{pg} / \mathrm{ml}$ & $200-1000 \mathrm{pg} / \mathrm{L}$ \\
Homocisteína* & $1905 \mathrm{pg} / \mathrm{ml}$ & \\
Lactato* & $92,6 \mu \mathrm{mol} / \mathrm{L}$ & $5-18 \mu \mathrm{mol} / \mathrm{L}$ \\
Piruvato & $3,04 \mathrm{nmol} / \mathrm{L}$ & $0,55-3,10 \mathrm{mmol} / \mathrm{L}$ \\
Amonio & $0,07 \mathrm{nmol} / \mathrm{L}$ & $0,03-0,08 \mathrm{nmol} / \mathrm{L}$ \\
Lactato/Piruvato & $45,2 \mu \mathrm{mol} / \mathrm{L}$ & $18-75 \mu \mathrm{mol} / \mathrm{L}$ \\
Orina & 43 & $<20-25$ \\
MMA* & & \\
MCA* & Elevado & Cualitativa \\
3-hidroxipropiónico & Elevado & Cualitativa \\
Postratamiento con vitamina B12 y ácido fólico & \\
Sangre & & Cualitatita \\
Homocisteína & $24,9 \mu \mathrm{mol} / \mathrm{L}$ & $20-80 \mu \mathrm{mol} / \mathrm{L} \neq$ \\
Vitamina B12 & $33,5 \mu \mathrm{mol} / \mathrm{L}$ & $200-1000 \mathrm{pg} / \mathrm{L}$ \\
\hline
\end{tabular}

C2: acetil-carnitina; C3: propionil-carnitina; C16: palmitoil-carnitina; C3/C2 propionil-carnitina/acetil-carnitina; $\mathrm{C} 3 / \mathrm{C} 16$ : propionil-carnitina/palmitoil-carnitina; MMA: ácido metilmalónico; MCA: ácido metilcítrico.

* Con correlación bioquímica según el fenotipo cblC. + No existe correlación bioquímica con fenotipo cblC. $¥$ Valores normales en pacientes tratados.

\section{DISCUSIÓN}

El diagnóstico inoportuno en este paciente hizo que las complicaciones neurológicas y hematológicas sean más graves, manifestadas como un retraso del desarrollo psicomotor severo, además de neutropenias recurrentes provocando múltiples hospitalizaciones. Además, nos mostró que las manifestaciones bioquímicas fueron diferentes con relación a los valores de vitamina B12, donde se describe que en la mayoría de los pacientes se encuentra normal. Planteamos que esta variante bioquímica se debe a que la mayoría de los niños descritos previamente se diagnostican en la etapa neonatal, y que la demora en el diagnóstico hizo que progresivamente los valores de esta vitamina aumenten notoriamente. Por la falta de una correlación bioquímica con algún error innato del metabolismo de la cobalamina, hizo que se planteara como parte de la terapéutica la inclusión de fórmulas especiales para la aciduria metilmalónica, y por las neutropenias se planteó el diagnóstico de inmunodeficiencia primaria. El diagnóstico de los TIC se basa utilizando la cromatografía de gases acoplada a espectrometría de masas en tándem o GC-MS ${ }^{[14]}$ de ácido metilmalónico en orina y plasma, así como de homocisteína; seguido de la determinación de las variantes patogénicas bialélicas de los diferentes grupos de complementación ${ }^{[7]}$. La GC-MS necesita de muestras congeladas, lo que hace complicada la conservación y el envío de la muestra. Sin embargo, lo que nos definió el diagnóstico fue el procesamiento de una muestra de sangre seca en papel filtro utilizando el secuenciamiento exómico clínico, facilitado por una mejor conservación y fácil envío. La respuesta bioquímica de la homocisteína al tratamiento estuvo dentro de lo esperado $(20-80 \mu \mathrm{mol} / \mathrm{L})^{[7]}$.

Tabla 2. Panorama actual del tamizaje neonatal universal en países de Latinoamérica.

\begin{tabular}{|c|c|c|c|c|c|c|}
\hline País & $\begin{array}{c}\text { Población } \\
\times 1000 \\
\text { hab }^{\mathrm{a}}\end{array}$ & $\begin{array}{l}\text { Nacimientos } \\
\times 1000 \text { hab }^{\mathrm{a}}\end{array}$ & Tamizaje inicial $^{b}$ & Tamizaje actual $^{b}$ & $\begin{array}{c}\text { Tecnología } \\
\text { utilizada }\end{array}$ & $\begin{array}{c}\text { Cobertura } \\
\text { (\%) }\end{array}$ \\
\hline Argentina & 43847 & 754 & 2006: PKU, CAH, CH, GAL, CF, BIO & 2011: PKU, CAH, CH, GAL, CF, BIO & Inmunoensayo & $>90$ \\
\hline Bolivia & 10888 & 253 & 2006: HC & $2006 \mathrm{HC}$ & Inmunoensayo & $\sim 20$ \\
\hline Brasil & 207653 & 2966 & 2001: PKU, HC, FQ, HP & 2014: PKU, CAH, CH, CF, BIO, Hbs & MS/MS & 85,8 \\
\hline Chile & 17910 & 239 & 1992: PKU, CH & 2015: PKU, CH, piloto + 25 & MS/MS & $>99$ \\
\hline Colombia & 48653 & 746 & 1999: CH & $\begin{array}{c}\text { 2019: PKU, CAH, CH, GAL, CF, BIO, } \\
\text { Hbs },+33\end{array}$ & MS/MS & $>80$ \\
\hline Ecuador & 16385 & 331 & 2011: PKU, CH, CAH, GAL & 2011: PKU, CAH, CH, GAL & Inmunoensayo & $>90$ \\
\hline México & 127540 & 2330 & 1995 & 2014: PKU, CAH, CH, CF, BIO & MS/MS & $>80$ \\
\hline Paraguay & 6725 & 140 & 2003: PKU, CH, CF & 2017: PKU, CAH, CH, GAL, CF, BIO & Inmunoensayo & $>75$ \\
\hline Perú & 31774 & 615 & 2012: PKU,CH,CAH,GAL,CF & 2019:PKU, CAH, CH, CF +2 & Inmunoensayo & $\sim 20$ \\
\hline Uruguay & 3444 & 49 & 1994: HC & 2013: PKU, CAH, CH, CF, +24 & MS/MS & $>99$ \\
\hline Venezuela & 31568 & 602 & 1999: PKU, HC & 1999: PKU, HC & Inmunoensayo & $\sim 70$ \\
\hline
\end{tabular}

a Datos obtenidos de UNICEF. El Estado Mundial de la Infancia 2017: Niños en un mundo digital. New York: UNICEF; 2017

${ }^{b}$ Adaptado de Therrell et al. Current status of newborn screening worldwide: 2015 . Program demographics and screened conditions in Latin American screening programs; y UNICEF. El Estado Mundial de la Infancia 2017: Niños en un mundo digital. New York: UNICEF; 2017

PKU: fenilcetonuria; HAC: hiperplasia adrenal congénita; $\mathrm{CH}$ : hipotiroidismo congénito; CF: fibrosis quística; GAL: galactosemia; BIO: déficit biotinasa; Hbs: hemoglobinopatías; MS/MS: espectrometría de masas en tándem. 
El tamizaje neonatal son programas de salud pública utilizadas mundialmente hace más de 50 años (estrategias de prevención secundaria), que se realizan a los recién nacidos asintomáticos y que no tienen un riesgo incrementado de algún problema de salud ${ }^{[15]}$. El principal objetivo es prevenir o mejorar a largo plazo consecuencias de alguna enfermedad potencialmente tratable ${ }^{[15]}$. En Perú, se tiene normado desde el 2019, el tamizaje neonatal de hipotiroidismo congénito, hiperplasia suprarrenal congénita, fenilcetonuria, fibrosis quística, hipoacusia congénita y catarata congénita ${ }^{[16]}$. Mientras que, en Latinoamérica el tamizaje neonatal se inicia desde los años 90s, llegando a tener países que realizan el tamizaje de hasta 29 condiciones (Tabla 2) ${ }^{[17,18]}$.

El presente caso nos muestra la importancia del tamizaje neonatal en las enfermedades potencialmente tratables con tecnologías más costo/efectivas que las utilizadas actualmente, como la espectrometría de masas en tándem ${ }^{[19]}$. Inclusive se han reportado resultados favorables por la metodología de secuenciamiento del gen MMACHC en muestras de vellosidades coriónicas de gestantes como parte del diagnóstico genético prenatal ${ }^{[20,21]}$.

El diagnóstico bioquímico de las acidurias orgánicas, en la mayoría de los casos se debe realizar a través de tecnologías como la cromatografía de masas en tándem en muestras de sangre y orina. Este tipo de pruebas no se encuentran disponible en nuestro medio, por lo que es de suma importancia tener convenios directos con laboratorios internacionales o mejor aún, implementar estas técnicas en al menos un centro de referencia, lo cual disminuiría notablemente los costos en el diagnóstico repercutiendo de manera directa en un manejo más efectivo. En tal sentido, las políticas públicas deberian establecer la prevalencia de estas entidades en estudios pilotos y según los resultados realizar su priorización en base a criterios ya establecidos desde hace mucho tiempo; no sólo con la intención de poder ofrecer tratamientos dirigidos, sino también con la finalidad de poder indicar el riesgo de recurrencia familiar y disminuir mediante tecnologías de reproducción asistida el número de niños con patologías devastadoras.

Contribuciones de autoría: Los autores declaran haber realizado contribuciones significativas a la concepción y diseño del manuscrito, la recolección de los datos, redacción y revisión crítica del contenido del manuscrito, la aprobación final de la versión que se publicará y asumen la responsabilidad frente a todos los aspectos del manuscrito.

Potenciales conflictos de interés: Los autores declaran no tener conflictos de intereses.

Fuente de financiamiento: Autofinanciado.

\section{ORCID:}

Hugo Hernán Abarca-Barriga: https://orcid.org/0000-0002-0276-2557 Richard S. Rodriguez: https://orcid.org/000-0002-0088-653X

\section{REFERENCIAS BIBLIOGRÁFICAS}

1. Garrod A. Inborns Errors of Metabolism: The Croonian Lectures delivered of Physician of London, in June 1908. JAMA. 1909;LIII(17):1427. doi: 10.1001/jama.1909.02550170083025

2. Stanton BF. New Understanding of Mechanisms and New Hope for Treatments. Pediatr Clin North Am. 2018;65(2):xvii-xviii. Doi: 10.1016/j.pcl.2018.01.002

3. Ferreira CR, van Karnebeek CDM, Vockley J, Blau N. A proposed nosology of inborn errors of metabolism. Genet Med Off J Am Coll Med Genet. 2019;21(1):102-6. doi: 10.1038/s41436-018-0022-8

4. Lerner-Ellis JP, Tirone JC, Pawelek PD, Doré C, Atkinson JL, Watkins $D$, et al. Identification of the gene responsible for methylmalonic aciduria and homocystinuria, cblC type. Nat Genet. 2006;38(1):93100. doi:10.1038/ng1683

5. Han B, Cao Z, Tian L, Zou H, Yang L, Zhu W, et al. Clinical presentation, gene analysis and outcomes in young patients with early-treated combined methylmalonic acidemia and homocysteinemia (cblC type) in Shandong province, China. Brain Dev. 2016;38(5):491-7. doi: 10.1016/j.braindev.2015.10.016

6. Lerner-Ellis JP, Anastasio N, Liu J, Coelho D, Suormala T, Stucki $\mathrm{M}$, et al. Spectrum of mutations in $M M A C H C$, allelic expression, and evidence for genotype-phenotype correlations. Hum Mutat. 2009;30(7):1072-81. doi: 10.1002/humu.21001

7. Sloan JL, Carrillo N, Adams D, Venditti CP. Disorders of Intracellular Cobalamin Metabolism. En: Adam MP, Ardinger HH, Pagon RA, Wallace SE, Bean LJ, Stephens K, et al., editors. GeneReviews ${ }^{\circledR}$ [Internet]. Seattle (WA): University of Washington, Seattle; 1993 [citado el 12 de agosto de 2020]. Disponible en: https://www. ncbi.nlm.nih.gov/books/NBK1116/

8. Andersson HC, Marble M, Shapira E. Long-term outcome in treated combined methylmalonic acidemia and homocystinemia. Genet Med Off J Am Coll Med Genet. 1999;1(4):146-50. doi:10.1097/00125817-199905000-00006

9. De Bie I, Nizard SDP, Mitchell GA. Fetal dilated cardiomyopathy: an unsuspected presentation of methylmalonic aciduria and hyperhomocystinuria, cblC type. Prenat Diagn. 2009;29(3):266-70. doi:10.1002/pd.2218

10. Carrillo-Carrasco N, Venditti CP. Combined methylmalonic acidemia and homocystinuria, cblC type. II. Complications, pathophysiology, and outcomes. J Inherit Metab Dis. 2012;35(1):103-14. doi:10.1007/s10545-011-9365-x

11. Rosenblatt DS, Aspler AL, Shevell MI, Pletcher BA, Fenton WA, Seashore MR. Clinical heterogeneity and prognosis in combined methylmalonic aciduria and homocystinuria (cblC). J Inherit Metab Dis. 1997;20(4):528-38. doi: 10.1023/a:1005353530303

12. Sharma AP, Greenberg CR, Prasad AN, Prasad C. Hemolytic uremic syndrome (HUS) secondary to cobalamin C (cblC) disorder. Pediatr Nephrol. 2007;22(12):2097-103. doi: 10.1007/s00467007-0604-1

13. Van Hove JLK, Van Damme-Lombaerts R, Grünewald S, Peters H, Van Damme B, Fryns J-P, et al. Cobalamin disorder Cbl-C presenting with late-onset thrombotic microangiopathy. Am J Med Genet. 2002;111(2):195-201. doi:10.1002/ajmg.10499

14. Ramsay J, Morton J, Norris M, Kanungo S. Organic acid disorders. Ann Transl Med. 2018;6(24):472. doi: 10.21037/atm.2018.12.39

15. Martínez-Morillo E, García BP, Menéndez FVÁ. Challenges for Worldwide Harmonization of Newborn Screening Programs. Clin Chem. 2016;62(5):689-98. doi: 10.1373/clinchem.2015.240903 
16. Resolución Ministerial N 558-2019/MINSA [Internet]. MINSA 2019 [citado el 12 de agosto de 2019]. Disponible en: https:// www.gob.pe/institucion/minsa/normas-legales/280743-5582019-minsa

17. Queiruga G. Neonatal screening - its importance and impact in Latin America. J Int Fed Clin Chem Lab Med. 2015;26(4):326331.

18. UNICEF. El Estado Mundial de la Infancia 2017: Niños en un mundo digital [Internet]. New York: UNICEF; 2017 [citado el 12 de agosto de 2019]. Disponible en: https://www.unicef.org/ media/48611/file
19. Feuchtbaum L, Cunningham G. Economic evaluation of tandem mass spectrometry screening in California. Pediatrics. 2006;117(5):S280-6. doi: 10.1542/peds.2005-2633G

20. Zong $\mathrm{Y}$, Liu $\mathrm{N}$, Zhao Z, Kong $\mathrm{X}$. Prenatal diagnosis using genetic sequencing and identification of a novel mutation in MMACHC. BMC Med Genet. 2015;16:48. doi:10.1186/s12881-015-0196-8

21. Hu S, Mei S, Liu N, Kong X. Molecular genetic characterization of cblC defects in 126 pedigrees and prenatal genetic diagnosis of pedigrees with combined methylmalonic aciduria and homocystinuria. BMC Med Genet. 2018;19(1):154. doi:10.1186/ s12881-018-0666-x 